\title{
Una propuesta metodológica para la medición de la sostenibilidad del turismo a escala local*
}

\author{
Javier Mendoza Jiménez \\ Raúl Hernández Martín \\ Universidad de La Laguna
}

\section{Resumen}

A pesar de la importancia creciente de la sostenibilidad en el turismo, su medición no cuenta todavía con una metodología con amplio consenso internacional, particularmente en el ámbito local. Este trabajo desarrolla una metodología para construir un sistema de indicadores sobre la sostenibilidad turística que incluye no solamente los aspectos internacionalmente comparables de la sostenibilidad sino también los aspectos específicos del destino. Como aspecto más significativo, se incorpora la dimensión territorial como un elemento fundamental para el análisis de la sostenibilidad, y se especifican los métodos para recoger las valoraciones de los distintos agentes.

Palabras clave: turismo y desarrollo, medición del turismo, sostenibilidad, planificación del desarrollo.

Clasificación JEL: O21, Q56, Z30, Z32.

\begin{abstract}
Despite of the growing importance of the sustainability in tourism, the measurement of this concept does not have yet a methodology with a broad international consensus, particularly at the local scale. This paper poses a methodology to create a set of indicator on tourism sustainability at a local level including not only the internationally comparable aspects of the sustainability but also the specific local aspects. As a most significant feature, the territorial dimension as a fundamental element for the analysis of the sustainability has been incorporated and the methods to receive assessments from different agents are specified.
\end{abstract}

Keywords: tourism and development, tourism data, sustainability, development planning.

JEL classification: O21, Q56, Z30, Z32.

\section{Introducción}

La definición más extendida de la sostenibilidad ha estado ligada a una asignación intergeneracional de las oportunidades y la satisfacción de las necesidades, tal y como se estableció desde hace tres décadas en el documento Nuestro Futuro Común (WCED, 1987). Sin embargo, a pesar del tiempo transcurrido y de la ingente investigación realizada, la conceptualización de la sostenibilidad no ha permitido establecer

* Este trabajo ha sido realizado en el contexto del proyecto de investigación Sistema de información para la medición de los impactos del turismo, con código 1808391702, financiado por la Fundación CajaCanarias y con el apoyo de la Unidad Mixta de Metodología e Investigación en Estadística Pública entre el Instituto Canario de Estadística y la Universidad de La Laguna. 
un marco ampliamente aceptado para su medición. Los fundamentos conceptuales de la sostenibilidad son, obviamente, externos a las investigaciones sobre el turismo (Buckley, 2012); por ello, es lógico pensar que el término turismo sostenible fue acuñado después de esa definición a nivel general y comparte algunas debilidades como la falta de un marco adecuado para su medición, a pesar de los avances realizados en el ámbito de la contabilidad ambiental (United Nations, 2014).

Así como la propia definición de la sostenibilidad turística y sus implicaciones ha sido objeto de un debate continuo, la medición de la sostenibilidad del turismo se ha visto dificultada tanto con la clarificación del propio objeto de análisis como con la dificultad de encontrar herramientas con las que dar respuesta a tan complejo y multidimensional problema. Ya desde los comienzos de la investigación sobre turismo y sostenibilidad se puso de manifiesto que la cuestión de la medición resultaba problemática (Liu, 2003), además, más recientemente McCool (2016) ha enfatizado la idea de que la indefinición de la escala de análisis, entre ellas la escala espacial, es uno de los factores que ayuda a explicar el atraso en la operativización de la sostenibilidad turística.

Entre los problemas principales para medir la sostenibilidad del turismo se encuentra que las escalas relevantes de análisis del fenómeno son tanto globales, como los aspectos relacionados con el cambio climático; nacionales y regionales, que es donde se concentra la capacidad de análisis y de intervención política; y la escala local, más relacionada con la gestión de los destinos (Hall, Gössling y Scott, 2015). Siguiendo a estos autores, los aspectos globales de la sostenibilidad son los que incorporan desafíos más novedosos en la gestión pública. Por su parte, la escala nacional ha centrado la atención desde la perspectiva de la conceptualización y la medición, como lo reflejan los esfuerzos metodológicos de United Nations, UNWTO, EUROSTAT y OECD (2010) y ha creado un marco para la traslación de conceptos y enfoques al ámbito regional. Sin embargo, la sostenibilidad del turismo a nivel local ha estado más vinculada al estudio de casos, sin un marco metodológico de análisis claro. Los esfuerzos metodológicos en este ámbito (WTO, 2004; European Union, 2016) han estado vinculados al desarrollo de baterías de indicadores cuantitativos que, aun siendo de gran utilidad, ofrecen una visión poco sistemática de sostenibilidad de los destinos.

En este artículo se aprovechan los trabajos previos para proponer una metodología para la medición de la sostenibilidad del turismo a nivel local que incluye tanto variables comunes, de relevancia internacional, como otras más específicas de cada territorio, de ámbito local. Esta metodología incorpora a los principales grupos de interés en la identificación y medición de la importancia de los factores clave, combinando indicadores objetivos con información basada en percepciones de los agentes. Igualmente, esta metodología incorpora de forma más precisa la dimensión territorial de los destinos turísticos como instrumento para posibilitar la medición y, a la vez, clarificar el objeto de análisis de la sostenibilidad del turismo a escala local.

Tras esta introducción, en el siguiente apartado se realiza una revisión de la literatura académica sobre la medición de la sostenibilidad turística, identificando las 
principales limitaciones encontradas. Más adelante se expondrá la metodología propuesta, a partir de la combinación de las diferentes aproximaciones metodológicas internacionales existentes y los procesos de selección de los factores, para la medición de la sostenibilidad del turismo. Posteriormente, se presentarán los resultados esperados para la metodología propuesta. Finaliza el trabajo con un apartado de conclusiones.

\section{La sostenibilidad del turismo y su medición}

La apertura del campo de investigación sobre el turismo sostenible se inicia con el debate en torno a conceptos como el ecoturismo (Cater y Lowman, 1994; Ziffer, 1989) y con el análisis de la relación entre dos conceptos inicialmente independientes, el turismo y el desarrollo sostenible (Nelson, Butler y Wall, 1993). Un enfoque crítico sobre dicha relación fue común en los primeros años, de modo que McKercher (1993) incluso planteaba si el turismo podría sobrevivir a la sostenibilidad debido a las políticas restrictivas para promoverla, lo cual ha sido rebatido recientemente por Pulido-Fernández, Andrades-Caldito y Sánchez-Rivero (2015). Por su parte, Butler (1990) criticaba la indefinición del término -algo que podría aplicarse más de 25 años después- y consideraba el concepto como una ficción y una pantalla de humo (Butler, 2015).

La relación entre turismo y sostenibilidad en la literatura académica ha adquirido especial relevancia a lo largo del siglo xxI. De este modo, el turismo, como una de las industrias más relevantes globalmente, ha sido relacionado con el cambio climático (Becken y Hay, 2007; Scott, Hall y Gössling, 2016), con la sobreexplotación de ciertos recursos naturales como el agua (Gössling, 2015) o las estrategias globales de crecimiento sostenible (Gössling, Ring, Dwyer, Andersson y Hall, 2016). Un claro indicador de la importancia del turismo para la sostenibilidad global es su inclusión dentro de los 17 Objetivos de Desarrollo Sostenible (United Nations, 2015) junto con los intentos, cada vez más frecuentes, de desarrollar una metodología para la medición de la sostenibilidad turística aceptada internacionalmente. De hecho, a pesar de dichos esfuerzos, los resultados para lograr un marco estadístico efectivo y comparable para la medición de la sostenibilidad turística han sido, hasta ahora, infructuosos como ponen de manifiesto Badenau et al. (2016) en su revisión de la literatura. La situación podría asemejarse a un puzle donde las piezas todavía no han logrado encajarse para formar una foto coherente, pero que cuenta con varias partes bien definidas. Por lo tanto, las dos preguntas enunciadas por Liu (2003) sobre cuál es el nivel sostenible de desarrollo del turismo y, sobre todo, cómo puede medirse dicho nivel, todavía no tienen respuesta.

Siguiendo a Bramwell, Higham, Lane y Miller (2017), el estudio de la sostenibilidad del turismo debería concentrarse con mayor frecuencia en entender y alterar las actitudes, comportamientos y elecciones de los actores individuales. Pero para ello sería preciso contar con indicadores que permitan evaluar la evolución de las 
diferentes dimensiones de la sostenibilidad a lo largo del tiempo. El enfoque de los indicadores objetivos ha sido mayoritario en la construcción de sistemas estadísticos para medir la sostenibilidad del turismo. Sin embargo, la falta de consenso sigue siendo un obstáculo no superado, como pone de manifiesto el trabajo de Tanguay, Rajaonson, Lefebvre y Lanoie (2010). Como señala McCool (2016), la medición, aunque importante, ha sido una cuestión dejada de lado en la discusión académica sobre la sostenibilidad del turismo.

La Organización Mundial del Turismo (en adelante UNWTO por sus siglas en inglés) ha impulsado una serie de iniciativas para definir los aspectos e indicadores del turismo más relevantes en el ámbito de la medición de su sostenibilidad. De este modo, en un primer esfuerzo se establecieron trece áreas divididas en secciones y a las que se asignan distintos indicadores (UNWTO, 2004). Otras dos iniciativas metodológicas internacionales sobre la medición de la sostenibilidad relevantes para el presente trabajo son el Sistema de Indicadores Europeo para los destinos sostenibles (ETIS por sus siglas en inglés) -que incluye 18 criterios centrados en la gestión del destino (European Union, 2016)- y el Sistema de Contabilidad Económica y Ambiental (SEEA) de Naciones Unidas, que tiene una perspectiva medioambiental y no está dirigido al ámbito turístico. No obstante, este último documento contiene definiciones y enfoques de gran interés para nuestra investigación, por ejemplo en el ámbito de la delimitación territorial (United Nations, 2014). Este trabajo plantea un esquema piramidal para las fases que van desde la obtención de información hasta la elaboración de indicadores clave, como puede verse en el Gráfico 1.

\section{GRÁFICO 1 \\ PIRÁMIDE DE LA INFORMACIÓN AMBIENTAL DEL SEEA}

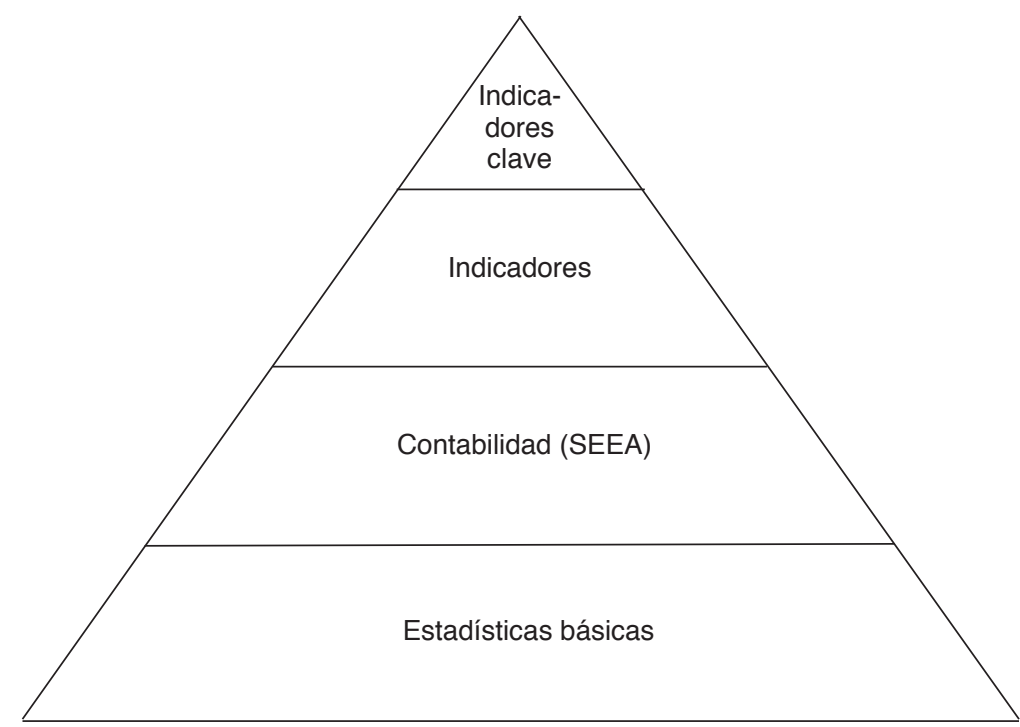

FUENTE: System of Environmental-Economic Accounting 2012 (United Nations, 2014). 
Una dificultad de estas propuestas metodológicas internacionales, así como de otras iniciativas de ámbito más local o regional, es la enorme cantidad de indicadores que emplean. A modo de ejemplo, en una iniciativa de este tipo para el caso de Andalucía se usan más de 300 indicadores (SAETA, 2007). Ello plantea la cuestión de las relaciones entre los indicadores, el peso o importancia de cada uno, los umbrales tolerables u óptimos así como la interpretación de sus variaciones. Este tipo de sistemas estadísticos están, además, basados mayoritariamente en indicadores cuantitativos si bien las percepciones, sobre todo a través de las opiniones de los distintos grupos de interés, han ido ganando en peso y relevancia. En relación a esto, Ko (2005) plantea que la dificultad de construir indicadores cualitativos es uno de los mayores obstáculos para medir el desarrollo sostenible y que, a falta de ellos, se les da demasiado peso a indicadores cuantitativos.

El desarrollo de indicadores cualitativos para la medición de la sostenibilidad del turismo ha tenido una cierta aceleración en los últimos años. Pérez et al. (2017) desarrollaron un sistema basado en la opinión de los distintos grupos de interés sobre los aspectos relevantes de la sostenibilidad. Igualmente, Randle y Hoye (2016) han usado una metodología similar en la definición del turismo sostenible en Australia, mientras que Poudel, Nyaupane y Budruk (2016) se basaron en la opinión de los grupos de interés para evaluar la sostenibilidad del turismo en áreas protegidas.

A pesar de las más de tres décadas de investigación en turismo sostenible, no existe todavía un consenso internacional ni en la definición del concepto ni en la metodología que debería usarse para su medición. Hasta el momento, el método más común ha sido el estudio de casos individuales, con un número escaso de estudios que incluyan varios destinos (Bramwell et al., 2017).

Esta falta de consenso es especialmente evidente si nos referimos al nivel local y subnacional, dado que la mayoría de los análisis y de las metodologías propuestas tienen como nivel de análisis los países o regiones. Una de las aportaciones de este trabajo es, precisamente, poner de manifiesto que las fronteras administrativas (nacionales, regionales o locales) no son un marco apropiado para la medición de la sostenibilidad, particularmente cuando se analiza la sostenibilidad de una actividad económica, puesto que esta normalmente no se adapta a esos límites artificiales.

El territorio ha sido poco considerado en la medición de la sostenibilidad. De hecho, el problema de la sostenibilidad (incluida la sostenibilidad turística) ha sido frecuentemente abordado desde un punto de vista global, proponiendo soluciones que en la mayoría de los casos no han tenido en cuenta las características distintivas de los territorios donde se pretendía analizar.

La Agenda Local 21 -a través de la cual las comunidades locales definen una estrategia de desarrollo sostenible y los programas de acción a implementar (UNEP y ICLEI, 2003)- fue un intento de introducir estas variables bajo la idea de pensar global, actuar local. Dicho enfoque tomaba en consideración los aspectos locales de la sostenibilidad, que tan relevantes son en el ámbito del turismo. Sin embargo, su impulso a partir de la Cumbre de Río no ha tenido continuidad debido a luchas de poder, una participación pública limitada y la fuerte influencia de la autoridad 
gubernamental dentro de las estructuras locales que pueden inhibir el desarrollo del turismo sostenible (Ruhanen, 2013).

Sobre la importancia de los factores locales, Inskeep (1991) señalaba que la sostenibilidad del turismo depende de cómo la planificación se adecúe a las características específicas del área. Desde este punto de vista ejecutivo, cuanto más pequeña sea la región identificable, más fácil será implementar estrategias de turismo sostenible (Miller y Twining-Ward, 2005).

La importancia de la sostenibilidad turística tiene, seguramente más que en otros sectores, una clara relación con la rentabilidad empresarial, no solamente desde la perspectiva de los costes (procesos más eficientes) sino de los ingresos (productos más atractivos). Por ello, la sostenibilidad está cada vez más asociada a la competitividad, existiendo investigaciones que evalúan la demanda de mercado para el turismo sostenible (Hardeman, Font y Nawijn 2017). De este modo, el concepto de destino sostenible o destino verde es cada vez más utilizado en estrategias promocionales.

Sin embargo, cabe preguntarse si un sistema universal de indicadores para la medición de la sostenibilidad del turismo es no solo posible sino deseable. Es obvio que existen factores comunes a todos los destinos pero no todos tienen la misma importancia en distintos lugares. Por tanto, la reciente tendencia de elaborar rankings donde se clasifica la sostenibilidad de los destinos en base a unos parámetros comunes deja fuera las características locales de los mismos que son fundamentales para entender y promover el desarrollo de un turismo sostenible (Mowforth y Munt, 2015). Un obstáculo para lograr definir mejor dichas características locales es la falta de desagregación de la información de muchos de los indicadores considerados como imprescindibles a nivel global.

De entre las iniciativas surgidas para abordar dicho problema, destaca la que propone la International Network on Regional Economics, Mobility and Tourism (INRouTe), que ha realizado avances en el diseño de un Sistema de Información de Turismo Regional (R-TIS), incluyendo una serie de guías para medir la relación entre el turismo y el desarrollo sostenible (INRouTe y UNWTO, 2012)

Asimismo, en 2016 la Organización Mundial del Turismo lanzó la iniciativa Measuring Sustainable Tourism (MST) con el objetivo de desarrollar un lenguaje común sobre la medición del turismo sostenible (UNWTO, 2016b). Uno de los retos que deben superarse para lograr ese lenguaje común en la medición de la sostenibilidad del turismo es la definición de las unidades territoriales relevantes de análisis. En este sentido, el trabajo de Hernández-Martín et al. (2016) introduce el concepto de micro-destino turístico basado en dos criterios principales: la concentración de establecimientos y las características de la oferta y tipologías del turismo. Se trata por lo tanto de un concepto que va más allá del puramente administrativo como podrían ser las regiones y los municipios, puesto que las fronteras de estas unidades 
administrativas no suelen coincidir con el área donde se desarrollan las actividades turísticas.

\section{Una propuesta metodológica}

En este apartado se exponen y fundamentan los criterios necesarios para la medición de la sostenibilidad del turismo a nivel local.

\subsection{Las variables a medir}

La propuesta metodológica que se presenta se alinea con la mencionada iniciativa MST de la UNWTO y se apoya en metodologías internacionales previamente reconocidas para construir un sistema estadístico. La primera cuestión que se aborda es el objetivo de la medición de la sostenibilidad. En este enfoque, la medición de la sostenibilidad de turismo es una herramienta para la toma de decisiones. Es decir, los conceptos de gobernanza y marco institucional deben ser considerados cuidadosamente, puesto que la medición no se hace al margen de consideraciones de política económica y turística. Si bien no existe una definición clara sobre lo que significa la gobernanza en el sector turístico (Durán-Fuentes, 2013), sí parece evidente que las conexiones y relaciones entre los distintos grupos de interés juegan un papel determinante en la definición y las acciones sobre la sostenibilidad.

Existe cierto consenso en considerar la sostenibilidad desde tres ángulos: económico, sociocultural y ambiental. Sin embargo, el hecho de que existan distintas definiciones de sostenibilidad condiciona determinar qué factores son los que hay que considerar exactamente (Ko, 2005). A fin de evitar ofrecer una batería de indicadores estadísticos inconexos y parciales, se ha optado por tomar como referencia el consenso internacional existente desde hace tiempo en torno a los temas de la agenda del turismo sostenible (UNEP y WTO, 2005). De este modo, siguiendo el objetivo de que esté orientado hacia la toma de decisiones, se ha optado por los 12 temas que componen dicha agenda para el turismo sostenible. El siguiente paso que debe definirse son las preguntas analíticas (UNWTO, 2016a), es decir, aquellas preguntas que el sistema para la medición de la sostenibilidad debería responder y que sirven de base para las futuras decisiones. El Cuadro 1 recoge una propuesta de preguntas que, posteriormente, debería adaptarse a los indicadores, realizada a partir de los 12 temas propuestos. 


\section{CUADRO 1}

\section{LAS VARIABLES DE LA SOSTENIBILIDAD TURÍSTICA Y LAS PREGUNTAS}

\begin{tabular}{|l|l|}
\hline 1. Viabilidad económica & ¿Es un destino competitivo? \\
\hline 2. Prosperidad local & $\begin{array}{l}\text { ¿Cuánto contribuye el turismo a la economía } \\
\text { local? }\end{array}$ \\
\hline 3. Calidad del empleo & $\begin{array}{l}\text { ¿Cuánto contribuye el turismo a la calidad del } \\
\text { empleo y al bienestar de los trabajadores? }\end{array}$ \\
\hline 4. Equidad social & $\begin{array}{l}\text { ¿Están los impactos del turismo sobre el bienestar } \\
\text { local repartidos de forma equilibrada? }\end{array}$ \\
\hline 5. Satisfacción del visitante & ¿Están los visitantes satisfechos con el destino? \\
\hline 6. Control local & $\begin{array}{l}\text { ¿Hasta qué punto está la población local in- } \\
\text { volucrada en la toma de decisiones públicas y } \\
\text { privadas sobre el turismo? }\end{array}$ \\
\hline 7. Bienestar de la comunidad & $\begin{array}{l}\text { ¿Cuánto está contribuyendo el turismo al biene- } \\
\text { star de la población local? }\end{array}$ \\
\hline 8. Riqueza cultural & $\begin{array}{l}\text { ¿Cómo está impactando el turismo en el patrimo- } \\
\text { nio cultural? }\end{array}$ \\
\hline 9. Integridad del territorio & $\begin{array}{l}\text { ¿Cuánta presión ejerce el turismo sobre el terri- } \\
\text { torio del destino? }\end{array}$ \\
\hline 10. Diversidad biológica & $\begin{array}{l}\text { ¿Cómo está afectando el turismo a la dotación de } \\
\text { recursos naturales? }\end{array}$ \\
\hline 11. Eficiencia de los recursos & $\begin{array}{l}\text { ¿Están siendo utilizados con eficiencia los recur- } \\
\text { sos escasos? }\end{array}$ \\
\hline 12. Pureza medioambiental & $\begin{array}{l}\text { ¿Cuál es la respuesta pública y privada a los } \\
\text { problemas medioambientales? }\end{array}$ \\
\hline
\end{tabular}

FUENTE: Elaboración propia a partir de UNEP y WTO (2005).

\subsection{Las unidades territoriales de análisis}

El turismo se ha definido tradicionalmente a través de dos perspectivas: la de demanda y la de oferta. La Cuenta Satélite del Turismo (UN, UNWTO, EUROSTAT y OECD, 2010) establece conceptos y definiciones para cada una de ellas. Desde el punto de vista de la demanda, la contribución económica del turismo debe ser aproximada desde las actividades de los turistas y su impacto en la adquisición de bienes y servicios. Por otro lado, la perspectiva de oferta presenta el turismo como un conjunto de actividades productivas que proveen principalmente a los visitantes o bien que son consumidas en su mayor parte por ellos (United Nations, 2016).

Sin embargo, una tercera perspectiva, la territorial, ha sido mucho menos desarrollada. La definición de los niveles de análisis espacial para el turismo es difusa, como prueba el hecho de que el destino turístico como tal todavía no cuente con una definición satisfactoria y ampliamente aceptada. 
El ya mencionado Sistema de Contabilidad Económica y Ambiental (en adelante SEEA por sus siglas en inglés) realiza una división en tres unidades de análisis: unidades espaciales básicas (BSU), unidad funcional de ecosistema (LCEU) y unidad de contabilidad de ecosistema (EAU). Esta clasificación ha sido utilizada como punto de partida en este trabajo a fin de realizar una propuesta de jerarquía entre las unidades de análisis, con el apoyo también en los trabajos previos realizados entre otros por Manning (1996) e INRouTe (2017).

De este modo, la unidad básica para esta clasificación es el establecimiento turístico, siguiendo la definición dada por United Nations et al. (2010). Dicha unidad tiene un paralelismo, además, a la definición que hace el SEEA de las unidades básicas como parcelas de tierra delimitadas por un catastro (United Nations, 2014).

Dichas unidades básicas pueden agruparse en un segundo nivel, que en este caso sería el destino turístico. Como se remarcó anteriormente, la definición de dicho concepto todavía no es clara a pesar de los esfuerzos realizados. Siguiendo a INRouTe (2017), el objeto de medida deben ser aquellas unidades territoriales donde el turismo es económicamente significativo. Ello se asocia con la concentración de establecimientos pertenecientes a actividades características del turismo cuya definición se recoge en United Nations (2016).

Esta segunda categoría no es una simple agregación de la primera, ya que incluye distintos productos y servicios turísticos al igual que otro tipo de recursos que no se incluyen dentro de los establecimientos turísticos. Para la definición de la última categoría, ha de tenerse en cuenta que, siguiendo el criterio de significatividad, no todas las áreas de una determinada región pueden ser calificadas como turísticas. Las áreas geográficas adyacentes a los destinos influyen y, especialmente, son influidas por el desarrollo turístico. Dichos espacios constituyen lo que se ha denominado como área turística de influencia (INRouTe, 2017), que podría ser definida, usando la analogía con el SEEA, como el ecosistema donde el turismo coexiste con el resto de actividades económicas, sociales y medioambientales. Otra cuestión diferente es la de los impactos indirectos del turismo, a través de las relaciones interempresariales, que sí se pueden difundir por todo el territorio del país en cuestión y de otros países (Badenau et al., 2016). Estos impactos afectan mayormente al enfoque global de la sostenibilidad y no tanto al enfoque local, tal y como serán definidos posteriormente.

Dichas áreas de influencia turística son de compleja delimitación, sobre todo en el caso de destinos continentales. Sin embargo, en algunas regiones, como serían las formadas por islas pequeñas, siguiendo las reflexiones entre otros de Carlsen y Butler (2011), la totalidad de la isla o la región podría adecuarse a esa definición de área de influencia turística.

En definitiva, la medición de la sostenibilidad turística a nivel de los destinos debe articularse en torno a tres unidades con diferente escala en el territorio, pero con evidentes interconexiones. Por un lado, el análisis de la sostenibilidad de los establecimientos turísticos, incluyendo sus procesos de producción; por otro lado, el análisis y la medición de la sostenibilidad de los destinos turísticos, como 
espacios de alta concentración de actividades turísticas, debidamente delimitados; y, finalmente, el análisis de las áreas de influencia turística, entendidas como zonas próximas a los destinos turísticos locales, que son frecuentemente visitadas por los turistas y que tienen relevancia en la medición de la sostenibilidad. Para identificar estas áreas de influencia pueden establecerse criterios de delimitación, por ejemplo, distancia respecto a los destinos turísticos, o bien seguir criterios administrativos más fáciles de aplicar, como sería por ejemplo un grupo de municipios, una comarca, una provincia o región, etc. Es importante destacar que esta delimitación de zonas no agota los impactos y la medición de la sostenibilidad del turismo puesto que, como se mencionó anteriormente, hay impactos directos que no suceden en los destinos y sus áreas de influencia, como suelen ser los derivados del transporte aéreo y, por otro lado, hay impactos indirectos del consumo y la producción turística que se difunden en el territorio a través de la cadena de proveedores y que tienen una localización bastante difusa en la geografía, incluyendo el propio país de destino, el país emisor y otros países proveedores de inputs a la cadena de valor turística.

\subsection{Enfoque global vs. enfoque local}

El turismo es indudablemente una industria global y como tal sus impactos también lo son. Por tanto, la medición de la sostenibilidad del turismo no puede estar referida solamente a los factores internos, sino que ha de considerarse también la influencia que los factores externos tienen en la unidad de análisis, y viceversa.

Partiendo de este argumento, en este trabajo se sugiere la existencia de tres enfoques diferenciados para la medición de la sostenibilidad del turismo, que son frecuentemente objeto de confusión, y que pueden verse resumidos en el Gráfico 2.

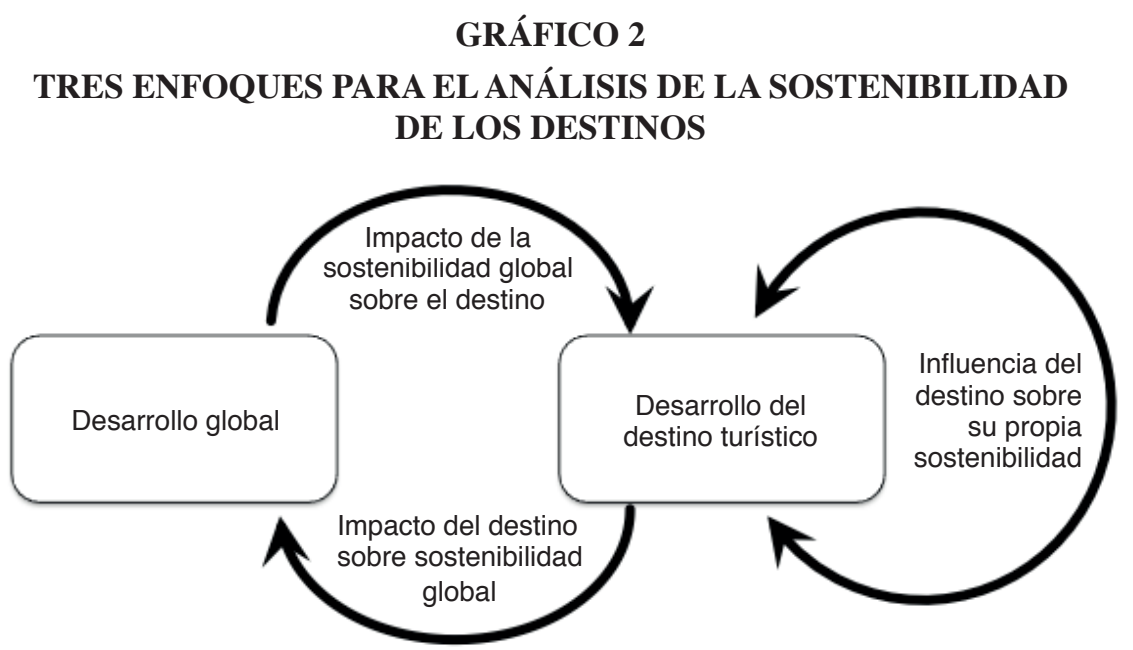

FUENTE: Elaboración propia. 
La primera aproximación se refiere al impacto del desarrollo turístico en un destino sobre la sostenibilidad global. En esta línea, el principal objeto de análisis ha sido el transporte -sobre todo aéreo- y los efectos que las emisiones de $\mathrm{CO}_{2}$ puedan tener (Becken, 2007; Becken y Hay, 2007; Gössling et al., 2007).

La segunda línea de análisis considera cómo la sostenibilidad global afecta al destino. Esto se relaciona principalmente con los estudios sobre los efectos del cambio climático sobre el turismo, donde el primero es percibido como una amenaza al segundo (Elsasser y Bürki, 2002; Gössling y Hall, 2006; Scott, Hall y Stefan, 2012).

La tercera categoría son los impactos del desarrollo turístico del destino sobre su propia sostenibilidad. Esta línea es la que mayor atención política ha atraído. Sin embargo, desde el punto de vista de la investigación académica aún hay mucho camino por recorrer en la medición de las consecuencias a largo plazo del desarrollo de los destinos sobre su propia viabilidad. La importancia de la medición del turismo desde esta perspectiva local es apoyada por McCool (2016), que señala que el nivel local no solo es donde el turismo ocurre sino donde se produce el desarrollo y las interacciones de los distintos actores. Esta importancia de la dimensión territorial exige la delimitación clara de las fronteras de los destinos turísticos. Por motivos de disponibilidad estadística y comodidad pueden aprovecharse fronteras administrativas como las municipales. Sin embargo, un análisis más detallado de la relación entre el turismo y el territorio nos llevaría a identificar los destinos turísticos como áreas de alta concentración de actividades características (INRouTe, 2017) y, por otro lado, las áreas de influencia que podrían incluir las zonas visitadas por los turistas, las zonas donde viven los trabajadores (UNEP y WTO, 205), etc.

\subsection{Los indicadores}

Para la construcción de un sistema estadístico se ha considerado en primer lugar el criterio de la comparabilidad. Este trabajo propone usar los doce aspectos para la sostenibilidad turística definidos anteriormente (UNEP y WTO, 2005) para crear un sistema de indicadores que sea comparable internacionalmente. A ello habría que añadir otros indicadores relevantes en el ámbito local objeto de estudio que tomen en consideración las características distintivas del destino (por ejemplo, la gestión de determinados recursos, situaciones sociales, desempleo, etc.) que puedan tener incidencia y relevancia particular en cada destino.

Los indicadores para la medición de la sostenibilidad deben ser de dos tipos. Por un lado, los indicadores objetivos, cuyos datos se extraen de mediciones de distintas variables, como podría ser la calidad del agua de una playa medida a través de parámetros químicos. Por otro lado, medición de la sostenibilidad debe también incorporar información basada en las percepciones de los agentes locales acerca del comportamiento de determinadas variables.

En el caso de este trabajo, los indicadores objetivos que se han tomado como base para el sistema estadístico provienen fundamentalmente de dos metodologías, 
siguiendo el diseño de la iniciativa MST a nivel global (UNWTO, 2016c). Estas dos metodologías centrales son el informe de la WTO (2004), donde se identifican 13 áreas principales - con sus correspondientes indicadores - y se señalan una serie de áreas claves a considerar, y el Sistema Europeo de Indicadores de Turismo (ETIS por sus siglas en inglés), que identifica 18 criterios que deben tenerse en cuenta para la gestión sostenible del destino (European Union, 2016). Complementariamente, se han considerado otras metodologías internacionales como el World Tourism Competitiveness Index (World Economic Forum, 2017), el Sistema Municipal de Indicadores de Sostenibilidad (Ministerio de Medioambiente y Medio Rural y Marino, 2010) y los estándares elaborados por Global Reporting Initiatives (2015). Estas metodologías incluyen un amplio catálogo de indicadores, quizás demasiado extenso, sin una ponderación en relación a su importancia, sin una interpretación clara en relación a la política turística y la sostenibilidad (por ejemplo, las variaciones de la estancia media no tienen una interpretación clara respecto a la sostenibilidad del turismo), sin un ámbito territorial de aplicación definido y con un sesgo hacia los indicadores cuantitativos. Es por ello que en nuestra propuesta se parte de una selección de los indicadores planteados en dichos documentos, pero se extenderá el análisis a otros indicadores locales relevantes y a las percepciones e importancia concedida por los stakeholders locales, tanto respecto a los grandes temas de la agenda de la sostenibilidad como a aquellos temas relevantes en la agenda local.

Después de un primer proceso de selección para evitar la repetición de indicadores, se realizó un proceso Delphi, basado en las recomendaciones de la WTO (2004), donde un grupo de expertos valoró de 1 a 5 los siguientes aspectos de los indicadores: relevancia, viabilidad, credibilidad, claridad, comparabilidad. El resultado incluye indicadores expresados en términos absolutos y otros en forma de tasas. Si bien estas últimas son preferibles para establecer comparaciones entre destinos, se incluyen también algunos indicadores en términos absolutos, que reflejan el tamaño del destino. El listado de indicadores propuesto no tiene una interpretación clara y directa en relación a la sostenibilidad del turismo y la toma de decisiones (que es la principal limitación de los sistemas basados en indicadores objetivos), pero en el contexto de otros indicadores objetivos locales y de las percepciones de los agentes sí pueden ser muy útiles. El resultado puede verse en el Cuadro 2.

Para la selección de las variables locales se plantea un proceso similar donde se aplica el método Delphi para la selección de un conjunto de variables que hayan sido enunciadas con anterioridad en documentos estratégicos del destino sobre la sostenibilidad. Obviamente, existirán algunas variables que serán comunes a muchos de los destinos, sin embargo, el objetivo de definirlas no es la comparabilidad, sino especificar los aspectos concretos en los que la gestión de la sostenibilidad debería centrarse para esa unidad territorial de análisis concreta.

Además de los indicadores objetivos, este estudio usa las percepciones de los grupos de interés. Este enfoque ha sido usado en numerosos estudios como una aproximación a la medida de la sostenibilidad (Mascarenhas, Nunes y Ramos, 2014; Poudel et al., 2016; Randle y Hoye, 2016), siendo los residentes el grupo que se ha 
CUADRO 2

INDICADORES PROPUESTOS PARA LA MEDICIÓN DE LA SOSTENIBILIDAD DEL TURISMO

\begin{tabular}{|c|c|c|}
\hline $\begin{array}{l}\text { Temas de sostenibilidad - } \\
\text { UNEP/UNWTO } 2005\end{array}$ & Indicadores propuestos & Metodología \\
\hline \multirow{3}{*}{ 1. Viabilidad económica } & Número de pernoctaciones por mes & ETIS \\
\hline & Estancia media de los visitantes (noches) & Otra \\
\hline & Rentabilidad hotelera (REVPAR) & Otra \\
\hline 2. Prosperidad local & $\begin{array}{l}\% \text { de los ingresos del turismo sobre el total de los } \\
\text { ingresos de la economía }\end{array}$ & WTO \\
\hline \multirow{3}{*}{ 3. Calidad del empleo } & $\begin{array}{l}\text { - Número de residentes empleados en turismo en } \\
\text { relación al total }\end{array}$ & WTO \\
\hline & $\begin{array}{l}\text { - Porcentaje del empleo directo del turismo sobre el } \\
\text { empleo total }\end{array}$ & ETIS \\
\hline & - \% de hombres y mujeres empleados en el turismo & ETIS \\
\hline 4. Equidad social & $\begin{array}{l}\text { - \% de residentes que consideran que el turismo ha } \\
\text { ayudado a realizar nuevos servicios e infraestructuras }\end{array}$ & WTO \\
\hline \multirow{2}{*}{ 5. Satisfacción del visitante } & - Nivel de satisfacción del visitante a su salida & WTO \\
\hline & $\%$ de visitantes repetidores & WTO \\
\hline 6. Control local & $\begin{array}{l}\text { - Percepción de participación de comunidades locales } \\
\text { en la planificación y toma decisiones }\end{array}$ & UNEP/WTO \\
\hline \multirow[t]{2}{*}{ 7. Bienestar de la comunidad } & $\begin{array}{l}\text { - Satisfacción de los residentes con el turismo (y con } \\
\text { componentes definidos del mismo) }\end{array}$ & WTO \\
\hline & Ratio entre $n .^{\circ}$ de visitantes/residentes (de media) & WTO \\
\hline 8. Riqueza cultural & $\begin{array}{l}\% \text { de residentes satisfechos con el impacto del tu- } \\
\text { rismo en la identidad cultural del destino }\end{array}$ & ETIS \\
\hline \multirow{2}{*}{ 9. Integridad física } & Grado de conservación de áreas naturales & Otra \\
\hline & $\%$ del territorio dedicado a actividades del turismo & Otra \\
\hline 10. Diversidad biológica & $\begin{array}{l}\mathrm{N} .{ }^{\circ} \text { de especies animales y vegetales presentes en el } \\
\text { destino (biodiversidad) }\end{array}$ & Otra \\
\hline \multirow{4}{*}{$\begin{array}{l}\text { 11. Eficiencia en el uso de } \\
\text { recursos }\end{array}$} & $\begin{array}{l}\text { - Consumo per cápita de energía de todas las fuentes } \\
\text { (general, del sector turístico por persona/día) }\end{array}$ & WTO \\
\hline & $\begin{array}{l}\text { - \% de la energía consumida proveniente de fuentes } \\
\text { renovables }\end{array}$ & WTO \\
\hline & $\begin{array}{l}\text { - Uso del agua (total consumido y litros por visitante/ } \\
\text { día) }\end{array}$ & WTO \\
\hline & - Ahorro de agua (\% reducido, recuperado o reciclado) & WTO \\
\hline \multirow{4}{*}{ 12. Pureza medioambiental } & $\begin{array}{l}\text { - \% de aguas residuales del destino que recibe tra- } \\
\text { tamiento }\end{array}$ & WTO \\
\hline & $\begin{array}{l}\text { - Volumen de residuos producido en el destino }(\mathrm{kg} \\
\text { por turista y día) }\end{array}$ & WTO \\
\hline & $\begin{array}{l}\text { - \% de residuos reciclados (especificado por frac- } \\
\text { ciones) }\end{array}$ & WTO \\
\hline & - Calidad del aire & Otra \\
\hline
\end{tabular}

FUENTE: Elaboración propia a partir de indicadores de WTO (2004), European Union (2016), UNEP y WTO (2005), World Economic Forum (2017), Ministerio de Medio Ambiente y Medio Rural y Marino (2010) y Global Reporting Initiative (2015). 
analizado más en profundidad. Las percepciones para construir indicadores se usan ya en las metodologías a las que nos hemos referido con anterioridad, por ejemplo, en indicadores relacionados con la calidad y la satisfacción. En esta propuesta se han tomado de nuevo como referencia los doce temas de la agenda de la sostenibilidad turística (UNEP y WTO, 2005) y sobre ellos se propone analizar las percepciones de los distintos grupos de interés a través de encuestas.

La ventaja de este sistema, como puede verse en el Gráfico 3, es que permite no solo conocer dichas percepciones, lo que supone una valiosa información para la toma de decisiones, sino que también permite hacer comparaciones en el tiempo, el espacio y entre las distintas categorías de grupos de interés.

Al problema de la definición de los instrumentos para la medición de la sostenibilidad (incluyendo la del turismo) se le une la indefinición existente sobre la importancia relativa de cada uno de los indicadores seleccionados. De este modo, la tendencia general suele ser considerar que todos los componentes de la medición de la sostenibilidad tienen la misma importancia, implantando de este modo una suerte de modelo general que no tiene en cuenta las características particulares de cada uno de los destinos analizados.

Por su parte, el establecimiento de los pesos de cada parte del sistema se ha resuelto de formas variadas. Así, Pulido-Fernández y Sánchez-Rivero (2009) y Peral, Lozano, Casas y Oyola (2010) resolvieron el problema a través de un análisis factorial. En el presente trabajo, cuyo objetivo no es ofrecer una visión sintética de la sostenibilidad turística, sino una visión panorámica de la misma que resulte útil para la toma de decisiones a partir de involucrar a los grupos de interés, se propone el uso del Importance Performance Analysis (en adelante IPA) como método para asignar la relevancia y pesos a cada uno de los indicadores definidos, tanto los comparables internacionalmente como los que son específicos del destino.

Siguiendo a Sever (2015), el IPA es una herramienta muy útil para diagnosticar las deficiencias y establecer prioridades en el desarrollo turístico, a fin de incrementar la satisfacción de los turistas y la competitividad de los destinos. Aunque presenta algunas limitaciones (Dwyer, Cvelbar, Edwards y Mihalic, 2012), el IPA ha sido considerado como un método robusto que puede aplicarse con relativa facilidad en estudios empíricos (Azzopardi y Nash, 2013). En cuanto al IPA aplicado a la sostenibilidad turística, pueden citarse entre otros los estudios de Sörensson y von Friedrichs (2013) o la experiencia de Ziegler, Dearden y Rollins (2012).

En este trabajo se propone seguir una metodología similar a la usada por Dwyer et al. (2014) en el estudio del turismo en Serbia. De este modo, se propone el diseño de encuestas para recabar la opinión de los grupos de interés sobre la importancia y satisfacción con el rendimiento de los factores locales que se han definido previamente y que se clasifican dentro de los 12 temas de la sostenibilidad turística ya mencionados.

El instrumento usado para dichas encuestas es una escala Lickert de 1 a 7 , que es uno de los métodos usados más frecuentemente en la investigación a través de encuestas (Awang, Afthanorhan y Mamat, 2016). Entre las ventajas que el uso del IPA 

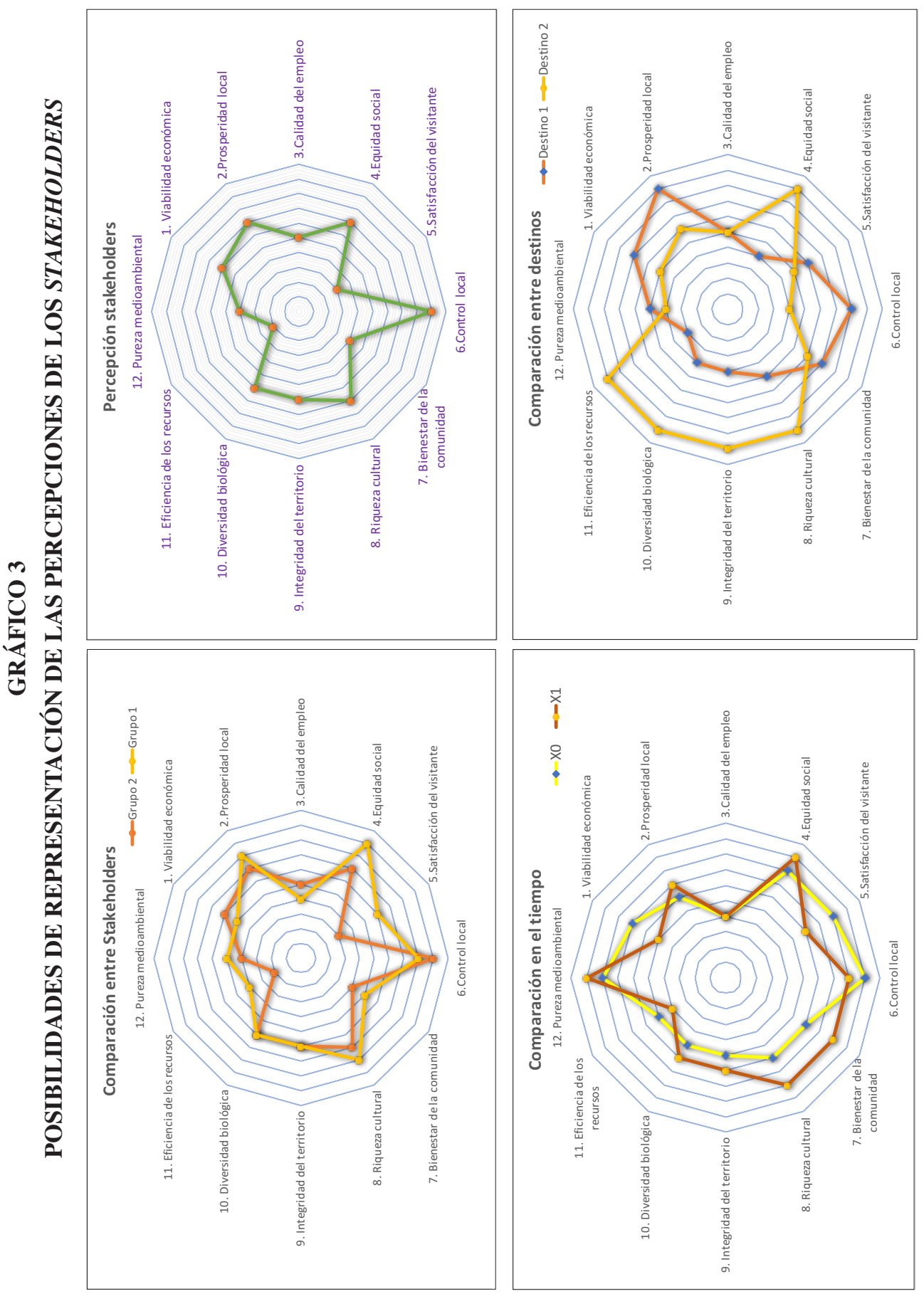

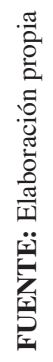


proporciona se encuentra la reducción de la subjetividad del investigador, el conformar un mapa sobre la opinión de los diferentes grupos de interés que luego puede ser desagregado en función de distintos criterios y el favorecer la comparación en el espacio y el tiempo, tanto dentro como fuera del destino analizado.

\section{Resultados esperados}

Teniendo en cuenta los planteamientos de los apartados anteriores, el análisis de la sostenibilidad turística no puede reducirse a un indicador sino que debe tomar como referencia un sistema en el que se combinen indicadores tanto objetivos como subjetivos (basados en las percepciones de distintos agentes). Igualmente, dicho sistema contiene indicadores con relevancia reconocida a nivel internacional junto con variables de interés en el destino, región o país turístico considerado. Es por ello que el sistema de indicadores propuesto no permite directamente conocer el grado o comparar la sostenibilidad entre destinos, pero sí constituye una herramienta para la toma de decisiones y para hacer comparaciones de algunas variables. El sistema de indicadores propuesto es una herramienta multidimensional que trata de sintetizar la información más relevante acerca de la sostenibilidad de un destino turístico de cara a la toma de decisiones.

Los resultados esperados del sistema de indicadores propuesto se presentan de forma sintética en el Cuadro 3.

El cuadrante superior izquierdo presenta un set de indicadores comparables internacionalmente identificados a partir del método Delphi y que sintetiza esfuerzos anteriores en este ámbito (WTO, 2004; European Union, 2016). Si bien la intención general de esta metodología no es establecer un sistema de clasificación entre destinos con respecto a la sostenibilidad, no es menos cierto que en la toma de decisiones

\section{CUADRO 3}

SISTEMA DE INDICADORES PARA LA MEDICIÓN DE LA SOSTENIBILIDAD DEL TURISMO

\begin{tabular}{|l|l|l|}
\hline \multicolumn{1}{|c|}{$\begin{array}{c}\text { Tipo de indicador/ } \\
\text { Escala de relevancia }\end{array}$} & \multicolumn{1}{|c|}{$\begin{array}{c}\text { Relevancia reconocida } \\
\text { internacional }\end{array}$} & \multicolumn{1}{|c|}{ Relevancia reconocida local } \\
\hline Indicadores objetivos & $\begin{array}{l}\text { Base en indicadores interna- } \\
\text { cionales de UNWTO (2004) }+ \\
\text { ETIS. } \\
\text { Lista consensuada por expertos } \\
\text { internacionales. }\end{array}$ & $\begin{array}{l}\text { Indicadores locales tomados } \\
\text { decumentos estratégicos. } \\
\text { stakeholders locales. por }\end{array}$ \\
\hline Indicadores de percepción & $\begin{array}{l}\text { IPA Valoración y relevancia } \\
\text { dada por los stakeholders lo- } \\
\text { cales sobre 12 agenda UNEP y } \\
\text { WTO (2005). }\end{array}$ & $\begin{array}{l}\text { IPA Desempeño y relevancia } \\
\text { por los stakeholders sobre va- } \\
\text { riables locales estratégicas. }\end{array}$ \\
\hline
\end{tabular}

FUENTE: Elaboración propia. 
es necesario conocer el posicionamiento de los competidores en algunos indicadores comparables internacionalmente. Tener una referencia que pueda ser utilizada para comparar destinos puede ayudar, además, a establecer estrategias de actuación en una escala mayor que el de las unidades territoriales de análisis más desagregadas.

El cuadrante superior derecho es similar al anterior, es decir, está basado en indicadores objetivos, pero centrado en aspectos característicos y diferenciales de la unidad territorial analizada. De este modo, a través de la consulta con los agentes locales, puede establecerse el nivel de importancia de cada uno de los aspectos y conocer en cuáles de ellos debe ponerse el foco de actuación al establecer las estrategias del destino.

El cuadrante inferior izquierdo complementa el primero de los cuadrantes analizados y ofrece una representación de las percepciones de los distintos grupos de interés acerca de la importancia y el desempeño del destino turístico respecto a los 12 temas de sostenibilidad tomados como referencia (UNEP y WTO, 2004). Se trata, por lo tanto, de una herramienta que permite no solo conocer cuál es la importancia que se le otorga y el desempeño percibido por parte de los stakeholders del destino en dichos aspectos, sino también realizar comparaciones entre temas y entre agentes que permitan identificar los aspectos clave que deben abordarse en la toma de decisiones política.

Por último, el cuadrante inferior derecho muestra un análisis de la importancia y la satisfacción con el rendimiento de las variables locales seleccionadas. Dicho análisis permitiría conocer si la distribución de los esfuerzos y recursos del destino en su estrategia es la correcta, así como adaptar las futuras estrategias a la situación resultante de la aplicación del IPA.

Estos resultados se obtendrán para los destinos turísticos locales, delimitados por fronteras administrativas (municipios) o por criterios de concentración turística (Hernández-Martín et al., 2016), así como para las áreas de influencia turística, que deben tener también unas fronteras bien definidas y que podrían corresponder a comarcas o incluso provincias.

En definitiva, la propuesta realizada aprovecha los sistemas de indicadores existentes para medir la sostenibilidad del turismo a nivel local, como United Nations (2004) o European Union (2016), pero también trata de superar algunas de sus limitaciones. Algunos de los problemas a los que se han enfrentado estas iniciativas y otras similares mencionadas anteriormente se relacionan con proporcionar baterías de indicadores sin ponderar su importancia y sin una clara relación con la política turística y con la sostenibilidad. Dichas iniciativas han carecido de una visión territorial clara, han tenido una cierta tendencia unificadora entre los destinos, dando escasa relevancia a las percepciones de los stakeholders locales y con un cierto sesgo hacia los indicadores cuantitativos. La propuesta que se realiza en este trabajo trata de contribuir a superar dichas debilidades y a aportar más valor y utilidad al esfuerzo de medición de la sostenibilidad del turismo a nivel local, siempre entendiendo la complejidad de este proceso y la inexistencia de soluciones sencillas que puedan resolver simultáneamente todos los desafíos que están incluidos dentro del 
objetivo general de medir la sostenibilidad del turismo a nivel local desde un enfoque orientado a la toma de decisiones.

\section{Conclusiones}

A pesar de la creciente importancia del turismo como campo de investigación en las últimas décadas, el análisis de la sostenibilidad turística (y especialmente su medición) no cuenta todavía con una metodología con un amplio consenso internacional que resulte operativa. Algunas valiosas iniciativas existentes han aportado resultados en los destinos que consideramos pueden ser mejorados si se incorpora de forma más clara un enfoque territorial que incluya la delimitación de los destinos locales, si se tiene más en cuenta las percepciones de diferentes tipos de stakeholders y su valoración sobre la importancia relativa de cada uno de los indicadores, si se reduce el número de indicadores cuantitativos utilizados, cuya utilidad e interpretación puede ser discutible y se tienen en cuenta, además de aspectos comunes a nivel internacional, los temas más específicos del destino local.

En este trabajo se ha propuesto una metodología en la que la sostenibilidad no se considera como una competición entre destinos ni puede reducirse a un único valor. Su medición no debe, por supuesto, ignorar la comparabilidad, siendo necesario establecer, en la medida de lo posible, parámetros comunes para todas las unidades territoriales de análisis. Sin embargo, los aspectos específicos locales deben tener importancia para una correcta definición y medición de la sostenibilidad del turismo. La propuesta que se hace combina las variables cuantitativas más relevantes a nivel internacional, que se seleccionan a través de una consulta a expertos, con las percepciones de diferentes agentes locales acerca de la sostenibilidad del turismo; así mismo, se incorporan nuevos indicadores objetivos de interés local que han sido identificados a través de los documentos estratégicos existentes en la zona. Además, la identificación clara de los límites de los destinos locales (no reduciendo lo local a los límites municipales) constituye otra aportación relevante, puesto que la consideración del territorio es fundamental para definir los impactos del turismo. La participación de los agentes en la medición de la sostenibilidad en un destino es también de capital importancia, especialmente por las orientaciones que se derivan para la implementación de políticas turísticas en los destinos.

Sin embargo, la aproximación realizada no resuelve, ni mucho menos, el problema de la medición de la sostenibilidad ni la conversión de estos indicadores objetivos y percepciones en medidas de política económica. No es sencillo traducir en políticas coherentes las valoraciones, a veces contradictorias, de los agentes acerca sobre los grandes temas de la agenda de la sostenibilidad o sobre los aspectos más específicos del destino. Tampoco aportan toda la información necesaria los indicadores objetivos consensuados internacionalmente, ni aquellos más específicos del destino. Es tan complejo el tema de análisis que tenemos que conformarnos con aproximaciones a la realidad que ofrezcan orientaciones y referencias a la política. 
La iniciativa MST que actualmente desarrolla la UNWTO debería ser un paso decidido para definir el necesario lenguaje común que permita establecer un marco de referencia para la medición de la sostenibilidad del turismo en las diferentes escalas de análisis, particularmente en la escala más local. La presente propuesta metodológica trata de contribuir a esta línea de trabajo, centrando el análisis en las consideraciones locales que son de gran importancia no solo para definir la sostenibilidad, sino también definir las estrategias para gestionarla.

\section{Referencias bibliográficas}

[1] AWANG, Z.; AFTHANORHAN, A. y MAMAT, M. (2016). «The Likert scale analysis using parametric based Structural Equation Modeling (SEM)». Computational Methods in Social Sciences, 4 (1), 13.

[2] AZZOPARDI, E. y NASH, R. (2013). «A critical evaluation of importance-performance analysis». Tourism Management, 35, 222-233.

[3] BUDEANU, A.; MILLER, G.; MOSCARDO, G. y OOI, C. S. (2016). «Sustainable tourism, progress, challenges and opportunities: an introduction». Journal of Cleaner Production, 111, 285-294.

[4] BECKEN, S. (2007). «Tourists' perception of international air travel's impact on the global climate and potential climate change policies». Journal of Sustainable Tourism, 15 (4), 351-368.

[5] BECKEN, S. y HAY, J. E. (2007). Tourism and climate change: Risks and opportunities (vol. 1). Channel View Publications, Clavedon.

[6] BRAMWELL, B.; Higham, J.; LANE, B. y MILLER, G. (2017). «Twenty-five years of sustainable tourism and the Journal of Sustainable Tourism: Looking back and moving forward». Journal of Sustainable Tourism, 25 (1), 1-9.

[7] BUCKLEY, R. (2012). «Sustainable tourism: Research and reality». Annals of Tourism Research, 39 (2), 528-546.

[8] BUTLER, R. (2015). «Sustainable Tourism: The Undefinable and Unachievable Pursued by the Unrealistic?», en T. V. Singh, Challenges in Tourism Research, Channel View Publications, Bristol.

[9] Butler, R. W. (1990). «Alternative tourism: Pious hope or Trojan horse? Journal of Travel Research, 28 (3), 40-45.

[10] CARLSEN, J. y BUTLER, R. (2011). Island Tourism: Towards a Sustainable Perspective. CABI, Wallingford.

[11] CATER, E. y LOWMAN, G. (1994). Ecotourism: A sustainable option? Wiley, Chichester.

[12] DURÁN-FUENTES, C. (2013). Governance for the Tourism Sector and its Measurement. UNWTO Issue Papers. Madrid.

[13] DWYER, L.; CVELBAR, L. K.; EDWARDS, D. y MIHALIC, T. (2012). «Fashioning a destination tourism future: The case of Slovenia». Tourism Management, 33 (2), 305316.

[14] DWYER, L.; DRAGIĆEVIĆ, V.; ARMENSKI, T.; MIHALIČ, T. y KNEŽEVIĆ CVELBAR, L. (2014). «Achieving destination competitiveness: an importanceperformance analysis of Serbia». Current Issues in Tourism, 19 (6), 1309-1336. 
[15] ELSASSER, H. y BÜRKI, R. (2002). «Climate change as a threat to tourism in the Alps». Climate Research, 20 (3), 253-257.

[16] (2016). The European Tourism Indicator System ETIS toolkit for sustainable destination management. European Commission.

[17] GLOBAL REPORTING INITIATIVE (2015). GRI G4 Guidelines 1 Reporting principles and standard disclosures. Global Reporting Initiative Board, Amsterdam.

[18] GÖSSLING, S. (2015). «New performance indicators for water management in tourism». Tourism Management, 46, 233-244.

[19] GÖSSLING, S.; BRODERICK, J.; UPHAM, P.; CERON, J.-P.; DUBOIS, G.; PEETERS, P. y StRASDAS, W. (2007). «Voluntary carbon offsetting schemes for aviation: Efficiency, credibility and sustainable tourism». Journal of Sustainable Tourism, 15 (3), 223-248.

[20] GÖSSLING, S. y HALL, C. M. (2006). «Uncertainties in predicting tourist flows under scenarios of climate change». Climatic Change, 79 (3-4), 163-173.

[21] GÖSSLING, S.; RING, A.; DWYER, L.; ANDERSSON, A.-C. y HALL, C. M. (2016). «Optimizing or maximizing growth? A challenge for sustainable tourism». Journal of Sustainable Tourism, 24 (4), 527-548.

[22] HALL, C. M.; GÖSSLING, S. y SCOTT, D. (2015). «Tourism and sustainability: an introduction». en C. M. Hall, S. Gössling y D. Scott (eds.), The Routledge Handbook of Tourism and Sustainability. Taylor \& Francis.

[23] HARDEMAN, G.; FONT, X. y NAWIJN, J. (2017). «The power of persuasive communication to influence sustainable holiday choices: Appealing to self-benefits and norms». Tourism Management, 59, 484-493.

[24] HERNÁNDEZ-MARTÍN, R.; SIMANCAS-CRUZ, M. R.; GONZÁLEZ-YANES, J. A.; RODRÍGUEZ-RODRÍGUEZ, Y.; GARCÍA-CRUZ, J. I. y GONZÁLEZ-MORA, Y. M. (2016). «Identifying micro-destinations and providing statistical information: a pilot study in the Canary Islands». Current Issues in Tourism, 19 (8), 771-790.

[25] INTERNATIONAL NETWORK ON REGIONAL ECONOMICS, MOBILITY AND TOURISM (2017). Tourism, territory and sustainability: a statistical insight at subnational level. Toward a set of UNWTO guidelines. Madrid.

[26] INSKEEP, E. (1991). Tourism planning: an integrated and sustainable development approach. Van Nostrand Reinhold, New York.

[27] INTERNATIONAL NETWORK ON REGIONAL ECONOMICS, MOBILITY AND TOURISM AND WORLD TOURISM ORGANIZATION (2012). A Closer Look at Tourism: Sub-national Measurement and Analysis-Towards a Set of UNWTO Guidelines. UNWTO, Madrid.

[28] KO, T. G. (2005). «Development of a tourism sustainability assessment procedure: a conceptual approach». Tourism Management, 26 (3), 431-445.

[29] LIU, Z. (2003). «Sustainable tourism development: A critique». Journal of Sustainable Tourism, 11 (6), 459-475.

[30] MANNING, T. (1996). What tourism managers need to know: A practical guide to the development and use of indicators of sustainable tourism. World Tourism Organization, Madrid.

[31] MASCARENHAS, A.; NUNES, L. M. y RAMOS, T. B. (2014). «Exploring the selfassessment of sustainability indicators by different stakeholders». Ecological Indicators, $39,75-83$. 
[32] McCOOL, S. F. y BOSAK, K. (2016). Reframing sustainable tourism. Springer, Dordrecht.

[33] McCOOL, S.F. (2016). «The changing meanings of sustainable tourism», en S. F. McCool y K. Bosak, Reframing sustainable tourism. Springer, Dordrecht.

[34] McKERCHER, B. (1993). «The unrecognized threat to tourism: Can tourism survive 'sustainability'?». Tourism Management, 14 (2), 131-136.

[35] MILLER, G. y TWINING-WARD, L. (2005). Monitoring for a sustainable tourism transition: The challenge of developing and using indicators. CABI, Wallingford.

[36] MINISTERIO DE MEDIO AMBIENTE Y MEDIO RURAL Y MARINO (2010). Sistema municipal de indicadores de sostenibilidad. Ministerio de Medio Ambiente y Medio Rural y Marino, Madrid.

[37] MOWFORTH, M. y MUNT, I. (2015). Tourism and sustainability: Development, globalisation and new tourism in the third world. Routledge, New York.

[38] NELSON, J.G.;BUTLER,R.y WALL,G.(1993).Tourismand sustainable development: monitoring, planning, managing. University of Waterloo Department of Geography.

[39] PERAL, F. J. B.; LOZANO, M. G.; CASAS, F. M. G. y OYOLA, M. L. (2010). «Indicadores sintéticos de turismo sostenible: una aplicación para los destinos turísticos de Andalucía».Rect@: Revista Electrónica de Comunicaciones y Trabajos de ASEPUMA, 11, 85-118.

[40] PÉREZ, V. E.; SANTOYO, A. H.; GUERRERO, F.; LEÓN, M. A.; SILVA, C. L. y CABALLERO, R. (2017). «Measuring the sustainability of Cuban tourism destinations considering stakeholders' perceptions». International Journal of Tourism Research, 19 (3), 318-328.

[41] POUDEL, S.; NYAUPANE, G. P. y BUDRUK, M. (2016). «Stakeholders’ perspectives of sustainable Tourism development: A new approach to measuring outcomes». Journal of Travel Research, 55 (4), 465-480.

[42] PULIDO FERNÁNDEZ, J. I. y SÁNCHEZ RIVERO, M. (2009). «Measuring tourism sustainability: proposal for a composite index». Tourism Economics, 15 (2), 277-296.

[43] PULIDO-FERNÁNDEZ, J. I.; ANDRADES-CALDITO, L. y SÁNCHEZ-RIVERO, M. (2015). «Is sustainable tourism an obstacle to the economic performance of the tourism industry? Evidence from an international empirical study». Journal of Sustainable Tourism, 23 (1), 47-64.

[44] RANDLE, E. J. y HOYE, R. (2016). «Stakeholder perception of regulating commercial tourism in Victorian National Parks, Australia». Tourism Management, 54, 138-149.

[45] RUHANEN, L. (2013). «Local government: facilitator or inhibitor of sustainable tourism development?». Journal of Sustainable Tourism, 21 (1), 80-98.

[46] SAETA,UNIDADDEANÁLISIS YESTADÍSTICADELTURISMO DEANDALUCÍA (2007). «Indicadores sobre turismo y sostenibilidad en los destinos: una aproximación desde Andalucía». Estudios Turísticos, 172-173, 131-139.

[47] SCOTT, D.; HALL, C. M. y GÖSSLING, S. (2016). «A review of the IPCC Fifth Assessment and implications for tourism sector climate resilience and decarbonization». Journal of Sustainable Tourism, 24 (1), 8-30.

[48] SCOTT, D.; HALL, C. M. y STEFAN, G. (2012). Tourism and climate change: Impacts, adaptation and mitigation. Routledge, New York.

[49] SEVER, I. (2015). «Importance-performance analysis: A valid management tool?» Tourism Management, 48, 43-53. 
[50] SÖRENSSON, A. y VON FRIEDRICHS, Y. (2013). «An importance-performance analysis of sustainable tourism: A comparison between international and national tourists». Journal of Destination Marketing \& Management, 2 (1), 14-21.

[51] TANGUAY, G. A.; RAJAONSON, J.; LEFEBVRE, J.-F. y LANOIE, P. (2010). «Measuring the sustainability of cities: An analysis of the use of local indicators». Ecological Indicators, 10 (2), 407-418.

[52] UNEP y ICLEI (2003). Tourism and Local Agenda 21, the role of local authorities in sustainable tourism.

[53] UNEP y WTO (2005). «Making tourism more sustainable». A Guide for Policy Makers. Paris and Madrid.

[54] UNITED NATIONS (2014). System of Environmental Economic Accounting 2012 Experimental Ecosystem Accounting. New York.

[55] UNITED NATIONS (2015). The 2030 Agenda for Sustainable Development. New York.

[56] UNITED NATIONS (2016). International Recommendations for Tourism Statistics 2008 Compilation Guide. United Nations Publications, New York.

[57] UNITED NATIONS, UNWTO, EUROSTAT y OECD (2010). Tourism Satellite Account: Recommended Methodological Framework 2008. New York.

[58] WTO (2004). Indicators of Sustainable Development for Tourism Destinations. A Guidebook. Madrid.

[59] UNWTO (2016a). Measuring Sustainable Tourism: Developing a statistical framework for sustainable tourism. Madrid.

[60] UNWTO (2016b). Measuring Sustainable Tourism (MST): Developing a statistical framework for sustainable tourism, Designing Pilot Studies. Madrid.

[61] UNWTO (2016c). Measuring Sustainable Tourism (MST): Developing a statistical framework for sustainable tourism - Framing Sustainable Tourism. Statistics and Tourism Satellite Account Programme. Madrid

[62] WCED (1987). Report of the World Commission on Environment and Development: Our Common Future. WCED.

[63] WORLD ECONOMIC FORUM (2017). The Travel \& Tourism Competitiveness Report 2017. World Economic Forum, Geneva.

[64] ZIEGLER, J.; DEARDEN, P. y ROLLINS, R. (2012). «But are tourists satisfied? Importance-performance analysis of the whale shark tourism industry on Isla Holbox, Mexico». Tourism Management, 33 (3), 692-701.

[65] ZIFFER, K. A. (1989). Ecotourism: The uneasy alliance. Conservation International, Washington. 